\title{
Solvability of a Hybrid Model for a Vertical Slender Structure ${ }^{1}$
}

\author{
A. Labuschagne ${ }^{2}$ and N. F. J. van Rensburg \\ Department of Mathematics and Applied Mathematics, \\ University of Pretoria, \\ Pretoria 0002, South Africa
}

\section{A. J. van der Merwe}

Department of Mechanical Engineering, Cape Peninsula University of Technology, Box 652, Cape Town 8000 South Africa

Received 20 January, 2008; accepted in revised form 30 November, 2009

\begin{abstract}
We consider the solvability of a hybrid model for the vibration of a vertical slender structure mounted on an elastic seating. The slender structure is modeled as a Rayleigh beam and gravity is taken into account. The seating and foundation block are modeled as rigid bodies connected by elastic springs with damping mechanisms. We show how an existence result for a general linear vibration problem in variational form may be applied to the weak variational problem for this system.
\end{abstract}

(C) 2009 European Society of Computational Methods in Sciences and Engineering

Keywords: Existence results, beam vibration, damping, hybrid model.

Mathematics Subject Classification: 74H20, 35G10, 47D06.

\section{Introduction}

Unwanted vibrations often occur in mechanical structures. Newland ([2], [3]) described the problem of wind-induced oscillations in vertical slender structures and explained the need for damping. To achieve this, "one method of artificially increasing the damping is to mount the chimney on a resilient foundation incorporating bearing pads made of a high-damping material."

Due to the complexity of such a composite structure, Newland proposed lumped parameter system models ([2], [3]). In [1] the vertical slender structure is modeled as an Euler-Bernoulli beam with rotary inertia (Rayleigh model) since Newland included rotary inertia. Apart from using beam theory, the models in [1] correspond to Newland's models in every respect - including the effect of gravity.

Modeling the behaviour of the resilient seating and foundation block leads to a hybrid system. In [1] it is shown that the interface conditions and additional equations can be accommodated in

${ }^{1}$ Published electronically December 10, 2009

${ }^{2}$ Corresponding author: anneke.labuschagne@up.ac.za 
the variational form. Consequently the finite element method can be used. Using a small number of elements, the results compared well with those of Newland (see [1], [2] and [3]). The numerical results published in [1] show clearly the advantage of the finite element method.

In this paper we consider the solvability of the most comprehensive of the hybrid models in [1]. For this purpose we consider a weak variational form of the model problem. This weak variational problem is expressed in terms of bilinear forms on suitable Hilbert spaces. The problem is to find $u$ with $u(t)$ in some Hilbert space $V$ for each $t>0$ and

$$
c\left(u^{\prime \prime}(t), v\right)+a\left(u^{\prime}(t), v\right)+b(u(t), v)=(f(t), v) \text { for each } v \in V,
$$

for specified $u(0)$ and $u^{\prime}(0)$. The bilinear form $c(.,$.$) is the inertia term, b(.,$.$) represents the$ elastic energy and the term $a(.,$.$) can accommodate various types of damping. The term on the$ right hand side represents forcing and $(.,$.$) denotes the inner product in the underlying Hilbert$ space. In a previous paper [4] an existence result for a general linear problem of the type above was proved.

The model problem is presented in Section 2 and in Section 3 it is written in dimensionless form. (The dimensionless form of the problem was considered in [1].) A Green Formula is presented in Section 4 with the derivation in the appendix. The main results on existence are presented in Section 5. Properties of the relevant product spaces are established in Section 6. (It should be noted that the weak variational form and properties of the product spaces are also required for error analysis in finite element applications.) Finally, the main results are proved in Section 7.

\section{Model problem}

The mathematical model is explained in [1], accompanied by sketches and free body diagrams. The vertical structure is modeled as a beam.

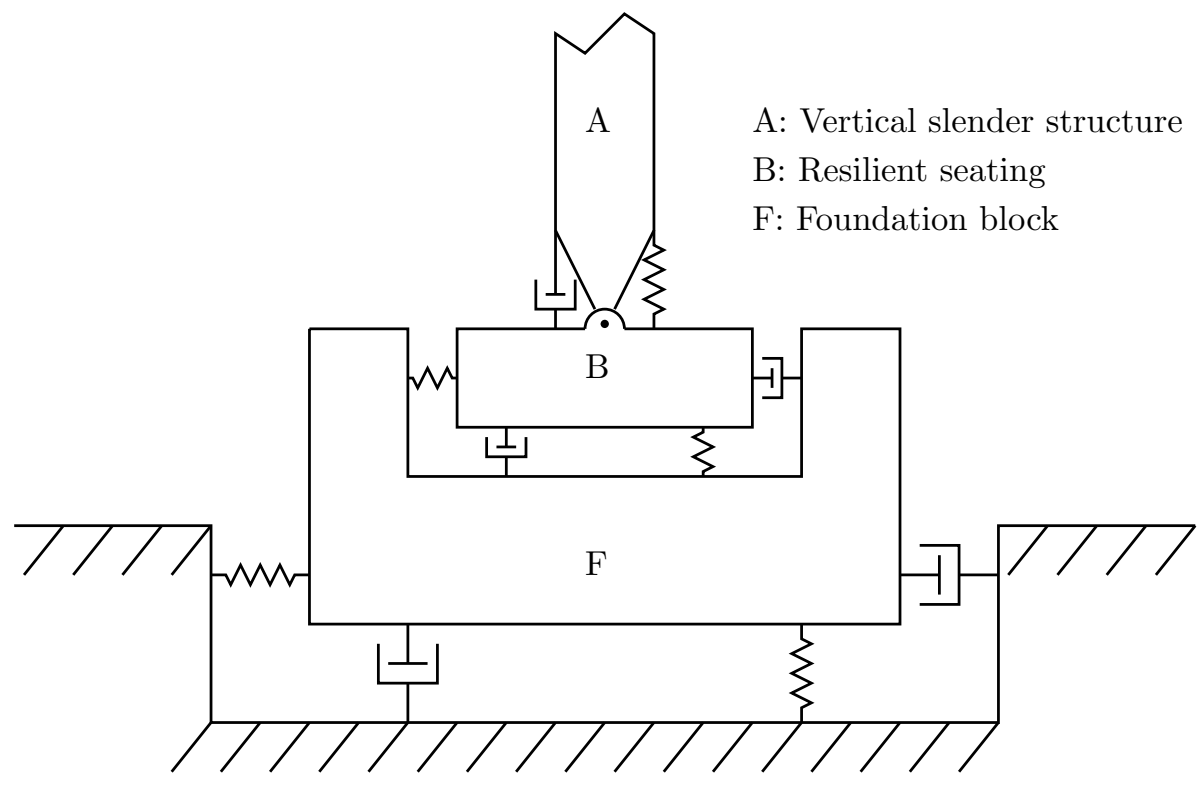

The beam is of length $\ell$ and has density $\rho$, cross sectional area $A$, area moment of inertia $I$ and Young's modulus $E$. Let the deflection (transverse displacement) of point $x$ in the reference configuration at time $t$ be denoted by $w(x, t)$. The equations of motion for the beam and the 
constitutive equations are given by Equations (1) to (4) below. In the equations, $V$ denotes the horizontal component of the force (shear force), $L$ a moment density due to gravity and $M$ the bending moment. The forcing term (due to the wind in this case) is denoted by $q$.

$$
\begin{aligned}
\rho A \partial_{t}^{2} w & =\partial_{x} V+q, \\
\rho I \partial_{t}^{2} \partial_{x} w & =\partial_{x} M+V+L, \\
M & =E I \partial_{x}^{2} w, \\
L(x, t) & =\rho A g(\ell-x) \partial_{x} w(x, t) .
\end{aligned}
$$

(These equations can be reduced to a single partial differential equation.)

The boundary conditions at $x=\ell$ follow from the fact that the shear force and bending moment at the top are zero:

$$
M(\ell, t)=V(\ell, t)=0 .
$$

To formulate interface conditions at the base $(x=0)$, it is necessary to derive equations of motion for the seating and foundation block (referred to as $B$ and $F$ in [1]). Both are modeled as rigid bodies connected to elastic springs and damping mechanisms. The interface conditions and the equations of motion of the foundation block and resilient seating reduce to the following five equations.

$$
\begin{aligned}
m_{B} \partial_{t}^{2} w(0, t)= & V(0, t)-K_{F B}\left(w(0, t)-w_{F}(t)\right) \\
& -C_{F B}\left(\partial_{t} w(0, t)-\dot{w}_{F}(t)\right), \\
M(0, t)= & k_{B A}\left(\partial_{x} w(0, t)-\theta_{B}(t)\right)+c_{B A}\left(\partial_{t} \partial_{x} w(0, t)-\dot{\theta}_{B}(t)\right), \\
I_{B} \ddot{\theta}_{B}(t)= & k_{B A}\left(\partial_{x} w(0, t)-\theta_{B}(t)\right)+c_{B A}\left(\partial_{t} \partial_{x} w(0, t)-\dot{\theta}_{B}(t)\right) \\
& -k_{F B}\left(\theta_{B}(t)-\theta_{F}(t)\right)-c_{F B}\left(\dot{\theta}_{B}(t)-\dot{\theta}_{F}(t)\right), \\
m_{F} \ddot{w}_{F}(t)= & K_{F B}\left(w(0, t)-w_{F}(t)\right)+C_{F B}\left(\partial_{t} w(0, t)-\dot{w}_{F}(t)\right) \\
& -K_{F} w_{F}(t)-C_{F} \dot{w}_{F}(t), \\
I_{F} \ddot{\theta}_{F}(t)= & k_{F B}\left(\theta_{B}(t)-\theta_{F}(t)\right)+c_{F B}\left(\dot{\theta}_{B}(t)-\dot{\theta}_{F}(t)\right) \\
& -k_{F} \theta_{F}(t)-c_{F} \dot{\theta}_{F}(t) .
\end{aligned}
$$

In these equations dots are used to indicate time derivatives, e.g. $\dot{w}_{F}=\frac{d}{d t} w_{F}$.

\section{Dimensionless form}

As mentioned in the introduction, we write the problem in terms of dimensionless variables. Let

$$
\xi=\frac{x}{\ell} \text { and } \tau=\frac{t}{T}
$$

The dimensionless displacement, forces and moments are chosen as

$$
\begin{gathered}
w^{*}(\xi, \tau)=\frac{w(x, t)}{\ell}, \quad V^{*}=\frac{\ell^{2} V(x, t)}{E I}, \quad q^{*}(\xi, \tau)=\frac{\ell q(x, t)}{E I}, \\
M^{*}(\xi, \tau)=\frac{\ell M(x, t)}{E I}, \quad L^{*}(\xi, \tau)=\frac{\ell^{2} L(x, t)}{E I}
\end{gathered}
$$

A convenient choice for $T$ is

$$
T=\ell^{2} \sqrt{\frac{\rho A}{E I}} .
$$

(C) 2009 European Society of Computational Methods in Sciences and Engineering (ESCMSE) 
We introduce dimensionless parameters for the beam:

$$
\alpha=\frac{\ell^{2} A}{I}, \quad \mu=\frac{\rho A \ell^{3} g}{E I} .
$$

Returning to the original notation, the Rayleigh beam model is given by

$$
\begin{aligned}
\partial_{t}^{2} w & =\partial_{x} V+q, \\
\frac{1}{\alpha} \partial_{t}^{2} \partial_{x} w & =\partial_{x} M+V+L, \\
M & =\partial_{x}^{2} w \\
L(x, t) & =\phi(x) \partial_{x} w(x, t) \text { with } \phi(x)=\mu(1-x) .
\end{aligned}
$$

(Note that this model contains only two parameters.)

The boundary conditions (5) at the top remain unaltered with $\ell=1$.

For the seating $\mathrm{B}$ and the foundation block $\mathrm{F}$ the dimensionless displacement, rotation, mass and moment of inertia are

$$
w^{*}(\tau)=\frac{w(t)}{\ell}, \quad \theta^{*}(\tau)=\theta(t), \quad m^{*}=\frac{m}{\ell \rho A}, \quad I^{*}=\frac{I}{\ell^{3} \rho A}
$$

and for the different elastic and damping constants

$$
K^{*}=\frac{K \ell^{3}}{E I}, \quad k^{*}=\frac{k \ell}{E I}, \quad C^{*}=\frac{C \ell^{3}}{E I T}, \quad c^{*}=\frac{c \ell}{E I T} .
$$

If we use the original notation to denote the dimensionless quantities, (6) to (10) remain unchanged.

\section{Green formula}

In this section we present a Green formula that will enable us to formulate a weak variational form for the hybrid vibration problem. This Green formula is also used to determine conditions for the solvability of the model problem. It is convenient to change to a product space formulation.

Define the product spaces $C=C[0,1] \times \mathbb{R}^{3}$ and $C^{k}=C^{k}[0,1] \times \mathbb{R}^{3}$.

For a given $u \in C^{4}, z \in C^{1}$ and $q \in C[0,1]$, consider $f \in C^{1}$ defined by

$$
\begin{aligned}
f_{1}= & V^{\prime}+q, \\
\frac{1}{\alpha} f_{1}^{\prime}= & M^{\prime}+V+L=u_{1}^{(3)}+V+\phi u_{1}^{\prime}, \\
V(1)= & 0 \\
m_{B} f_{1}(0)= & V(0)-K_{F B}\left(u_{1}(0)-u_{3}\right)-C_{F B}\left(z_{1}(0)-z_{3}\right), \\
I_{B} f_{2}= & k_{B A}\left(u_{1}^{\prime}(0)-u_{2}\right)+c_{B A}\left(z_{1}^{\prime}(0)-z_{2}\right) \\
& -k_{F B}\left(u_{2}-u_{4}\right)-c_{F B}\left(z_{2}-z_{4}\right), \\
m_{F} f_{3}= & K_{F B}\left(u_{1}(0)-u_{3}\right)+C_{F B}\left(z_{1}(0)-z_{3}\right)-K_{F} u_{3}-C_{F} z_{3}, \\
I_{F} f_{4}= & k_{F B}\left(u_{2}-u_{4}\right)+c_{F B}\left(z_{2}-z_{4}\right)-k_{F} u_{4}-c_{F} z_{4} .
\end{aligned}
$$

We use the notation $(f, g)=\int_{0}^{1} f g$ and $\|f\|=\sqrt{(f, f)}$. 


\section{Proposition 1}

Suppose $u \in C^{4}, z \in C^{1}$ and $q \in C[0,1]$. Then $f$ is well-defined by Equations (15) - (21), $f \in C^{2}$ and

$$
\begin{aligned}
& \left\|f_{1}\right\|^{2}+\frac{1}{\alpha}\left\|f_{1}^{\prime}\right\|^{2}+m_{B}\left(f_{1}(0)\right)^{2} \\
= & -\left(u_{1}^{(4)}+\left(\phi u_{1}^{\prime}\right)^{\prime}-q, f_{1}\right)+\left(u_{1}^{(3)}(1)+\phi(1) u_{1}^{\prime}(1)\right) f_{1}(1) \\
& +\left(-u_{1}^{(3)}(0)-\phi(0) u_{1}^{\prime}(0)-K_{F B}\left(u_{1}(0)-u_{3}\right)-C_{F B}\left(z_{1}(0)-z_{3}\right)\right) f_{1}(0) .
\end{aligned}
$$

Proof See the appendix.

\section{Remark}

By doing substitutions into Equations (15) - (23) according to the following table, we obtain the original model problem.

\begin{tabular}{|c|c|c|c|}
\hline$u_{1}$ & $u_{2}$ & $u_{3}$ & $u_{4}$ \\
$w(\cdot, t)$ & $\theta_{B}(t)$ & $w_{F}(t)$ & $\theta_{F}(t)$ \\
\hline$z_{1}$ & $z_{2}$ & $z_{3}$ & $z_{4}$ \\
$\partial_{t} w(\cdot, t)$ & $\dot{\theta}_{B}(t)$ & $\dot{w}_{F}(t)$ & $\dot{\theta}_{F}(t)$ \\
\hline$f_{1}$ & $f_{2}$ & $f_{3}$ & $f_{4}$ \\
$\partial_{t}^{2} w(\cdot, t)$ & $\ddot{\theta}_{B}(t)$ & $\ddot{w}_{F}(t)$ & $\ddot{\theta}_{F}(t)$ \\
\hline
\end{tabular}

To obtain the Green formula, we need two additional boundary conditions:

$$
\begin{aligned}
& u_{1}^{\prime \prime}(1)=M(1)=0 \\
& u_{1}^{\prime \prime}(0)=M(0)=k_{B A}\left(u_{1}^{\prime}(0)-u_{2}\right)+c_{B A}\left(z_{1}^{\prime}(0)-z_{2}\right),
\end{aligned}
$$

To formulate the Green formula, we introduce the following notation.

$$
\begin{aligned}
& c_{A}(f, v)=\left(f_{1}, v_{1}\right)+\frac{1}{\alpha}\left(f_{1}^{\prime}, v_{1}^{\prime}\right)+m_{B} f_{1}(0) v_{1}(0), \\
& b_{A}(u, v)=\left(u_{1}^{\prime \prime}, v_{1}^{\prime \prime}\right)-\left(\phi u_{1}^{\prime}, v_{1}^{\prime}\right) .
\end{aligned}
$$

The bilinear forms $a, b$ and $c$ are defined as follows.

$$
\begin{aligned}
c(f, v)= & c_{A}(f, v)+I_{B} f_{2} v_{2}+m_{F} f_{3} v_{3}+I_{F} f_{4} v_{4}, \\
a(z, v)= & C_{F B}\left(z_{1}(0)-z_{3}\right)\left(v_{1}(0)-v_{3}\right)+c_{B A}\left(z_{1}^{\prime}(0)-z_{2}\right)\left(v_{1}^{\prime}(0)-v_{2}\right) \\
& +c_{F B}\left(z_{2}-z_{4}\right)\left(v_{2}-v_{4}\right)+C_{F} z_{3} v_{3}+c_{F} z_{4} v_{4}, \\
b(u, v)= & b_{A}(u, v)+K_{F B}\left(u_{1}(0)-u_{3}\right)\left(v_{1}(0)-v_{3}\right) \\
& +k_{B A}\left(u_{1}^{\prime}(0)-u_{2}\right)\left(v_{1}^{\prime}(0)-v_{2}\right)+k_{F B}\left(u_{2}-u_{4}\right)\left(v_{2}-v_{4}\right) \\
& +K_{F} u_{3} v_{3}+k_{F} u_{4} v_{4} .
\end{aligned}
$$

\section{Proposition 2 (Green formula)}

Suppose $u \in C^{4}, z \in C^{1}$ and $q \in C[0,1]$ is given. If $f$ is defined by Equations (15) - (21) and $u$ and $z$ satisfy Equations (22) and (23), then

$$
c(f, v)+a(z, v)+b(u, v)=\left(q, v_{1}\right) \text { for each } v \in C^{2} .
$$

Proof See the appendix.

\section{Remark}

If we substitute according to the table into (24), we obtain the variational form for the model problem. This variational form may be used for finite element method approximations. 


\section{Existence}

To investigate the solvability of the model problem, we consider a weak variational form in suitable product spaces. Let

$$
X=\mathcal{L}^{2}(0,1) \times \mathbb{R}^{3} \text { and } H^{k}=H^{k}(0,1) \times \mathbb{R}^{3},
$$

where $H^{k}(0,1)$ denotes a Sobolev space. For the space $X$ we use the inner product

$$
(f, g)_{X}=\left(f_{1}, g_{1}\right)+f_{2} g_{2}+f_{3} g_{3}+f_{4} g_{4} .
$$

Note that the bilinear forms $a$ and $b$ are defined on $H^{2}$ and $c$ is defined on $H^{1}$.

Problem WVP

Given $u^{0} \in H^{2}, u^{+} \in H^{2}$ and $g \in C((0, \infty) ; X)$, find $u$ such that, for each $t>0, u(t) \in H^{2}$, $u^{\prime}(t) \in H^{2}, u^{\prime \prime}(t) \in H^{1}$ and

$$
\begin{gathered}
c\left(u^{\prime \prime}(t), v\right)+a\left(u^{\prime}(t), v\right)+b(u(t), v)=(g(t), v)_{X} \text { for each } v \in H^{2}, \\
\text { with } u(0)=u^{\circ} \text { and } u^{\prime}(0)=u^{+} .
\end{gathered}
$$

Note that Problem WVP is the weak variational form of the hybrid vibration problem if $g_{1}(t)=$ $q(\cdot, t)$ and $g_{2}=g_{3}=g_{4}=0$.

To prove existence for Problem WVP, we assume the following set of inequalities for the parameters.

\section{Stability condition}

$$
\frac{\rho A \ell^{3} g}{E I}<\frac{1}{2}, \quad k_{B A}>4 \rho A \ell^{2} g, \quad k_{F B}>8 \rho A \ell^{2} g \text { and } k_{F}>8 \rho A \ell^{2} g,
$$

or equivalently, in dimensionless form

$$
2 \mu<1, \quad k_{B A}^{*}>4 \mu, \quad k_{F B}^{*}>8 \mu \text { and } k_{F}^{*}>8 \mu .
$$

The inequalities on the parameters guarantee that the stiffness of the beam and springs are sufficient to ensure stability of the equilibrium. These conditions are physically realistic and will only be violated for a structure with excessive length or density (see also [1]).

\section{Theorem 1}

\section{Suppose}

(a) the stability condition holds,

(b) $u^{\circ} \in H^{2}, u^{+} \in H^{2}$ and there exists a $y \in H^{1}$ such that

$$
b\left(u^{\circ}, v\right)+a\left(u^{+}, v\right)+c(y, v)=0 \text { for all } v \in H^{2} .
$$

Then Problem WVP has a unique solution for each $g \in C^{1}([0, \infty), X)$. In addition

$$
u \in C\left([0, \infty), H^{2}\right) \cap C^{1}\left((0, \infty), H^{2}\right) \cap C^{1}\left([0, \infty), H^{1}\right) \cap C^{2}\left((0, \infty), H^{1}\right) .
$$

Proof See Section 7.

\section{Remark}

Condition (b) may be questioned. In Section 7 this condition is related to semigroup theory and Theorem 2 below provides sufficient conditions for (b) to hold. 


\section{Theorem 2}

Suppose $u \in H^{4}$ and $z \in H^{2}$. If $u$ and $z$ satisfy (22) and (23), i.e.

$$
\begin{aligned}
& u_{1}^{\prime \prime}(1)=M(1)=0 \\
& u_{1}^{\prime \prime}(0)=M(0)=k_{B A}\left(u_{1}^{\prime}(0)-u_{2}\right)+c_{B A}\left(z_{1}^{\prime}(0)-z_{2}\right),
\end{aligned}
$$

then there exists a unique $f \in H^{1}$ such that

$$
c(f, v)+a(z, v)+b(u, v)=0 \text { for each } v \in H^{2} .
$$

Proof See Section 7.

\section{$6 \quad$ Estimates}

The inner product for $X$ is defined in the previous section. The norm on $\mathcal{L}^{2}[0,1]$ is denoted by $\|\cdot\|$ and the induced norm on $X$ by $\|\cdot\|_{X}$.

The inner products of $H^{k}(0,1)$ and $\mathbb{R}^{3}$ can be used to define the obvious inner products for the product space $H^{k}$. The corresponding norm for $H^{k}$ is denoted by \|\|$_{H^{k}}$, while the norm for the Sobolev space $H^{k}(0,1)$ is denoted by $\|.\|_{k}$.

We also require a trace operator $\gamma$. If $\gamma u=u(0)$ for $u \in C^{1}[0,1]$, then $\gamma$ can be extended to $H^{1}(0,1)$.

It is clear that the symmetric bilinear form $c$ is an inner product for $H^{1}$. We proceed to show that the symmetric bilinear form $b$ is an inner product for the space $H^{2}$ provided that the stability condition holds for the parameters of the problem.

\section{Proposition 3}

If the stability condition holds, then there exists a constant $K_{b c}$ such that

$$
c(u, u) \leq K_{b c} b(u, u) \text { for each } u \in H^{2} .
$$

\section{Proof}

We use the elementary inequalities $\|x\| \leq\|x-y\|+\|y\|$ and $(a+b)^{2} \leq 2\left(a^{2}+b^{2}\right)$ as well as the the Poincaré inequality,

$$
\left\|u_{1}\right\| \leq\left\|u_{1}^{\prime}\right\|+\left|\gamma u_{1}\right| \text { for } u_{1} \in H^{1}(0,1) .
$$

This implies that $\left\|u_{1}\right\|^{2} \leq 2\left\|u_{1}^{\prime}\right\|^{2}+2\left(\gamma u_{1}\right)^{2}$ and $\left\|u_{1}^{\prime}\right\|^{2} \leq 2\left\|u_{1}^{\prime \prime}\right\|^{2}+2\left(\gamma u_{1}^{\prime}\right)^{2}$. Therefore

$$
c_{A}(u, u) \leq 2\left(2+\frac{1}{\alpha}\right)\left(\left\|u_{1}^{\prime \prime}\right\|^{2}+\left(\gamma u_{1}^{\prime}\right)^{2}\right)+\left(2+m_{B}\right)\left(\gamma u_{1}\right)^{2} .
$$

As $\left(\gamma u_{1}\right)^{2} \leq 2\left(\gamma u_{1}-u_{3}\right)^{2}+2 u_{3}^{2},\left(\gamma u_{1}^{\prime}\right)^{2} \leq 2\left(\gamma u_{1}^{\prime}-u_{2}\right)^{2}+4\left(u_{2}-u_{4}\right)^{2}+4 u_{4}^{2}$ and $u_{2}^{2} \leq\left(u_{2}-u_{4}\right)^{2}+u_{4}^{2}$, it follows that

$$
\begin{aligned}
c(u, u) & =c_{A}(u, u)+I_{B} u_{2}^{2}+m_{F} u_{3}^{2}+I_{F} u_{4}^{2} \\
& \leq K_{c}\left(\left\|u_{1}^{\prime \prime}\right\|^{2}+\left(\gamma u_{1}^{\prime}-u_{2}\right)^{2}+\left(\gamma u_{1}-u_{3}\right)^{2}+\left(u_{2}-u_{4}\right)^{2}+u_{3}^{2}+u_{4}^{2}\right)
\end{aligned}
$$

From the fact that

$$
\int_{0}^{1}(1-x)\left(u_{1}^{\prime}(x)\right)^{2} d x \leq\left\|u_{1}^{\prime}\right\|^{2},
$$

and using the inequality for $\left(\gamma u_{1}^{\prime}\right)^{2}$ above, it follows that

$$
\begin{aligned}
b_{A}(u, u) & =\left\|u_{1}^{\prime \prime}\right\|^{2}-\mu \int_{0}^{1}(1-x)\left(u_{1}^{\prime}(x)\right)^{2} d x \\
& \geq\left\|u_{1}^{\prime \prime}\right\|^{2}-\mu\left\|u_{1}^{\prime}\right\|^{2} \\
& \geq(1-2 \mu)\left\|u_{1}^{\prime \prime}\right\|^{2}-\mu\left(4\left(\gamma u_{1}^{\prime}-u_{2}\right)^{2}+8\left(u_{2}-u_{4}\right)^{2}+8 u_{4}^{2}\right) .
\end{aligned}
$$

(C) 2009 European Society of Computational Methods in Sciences and Engineering (ESCMSE) 
Consequently,

$$
\begin{aligned}
b(u, u) \geq & (1-2 \mu)\left\|u_{1}^{\prime \prime}\right\|^{2}+K_{F B}\left(\gamma u_{1}-u_{3}\right)^{2}+\left(k_{B A}-4 \mu\right)\left(\gamma u_{1}^{\prime}-u_{2}\right)^{2} \\
& +\left(k_{F B}-8 \mu\right)\left(u_{2}-u_{4}\right)^{2}+K_{F} u_{3}^{2}+\left(k_{F}-8 \mu\right) u_{4}^{2} \\
\geq & K_{b}\left(\left\|u_{1}^{\prime \prime}\right\|^{2}+\left(\gamma u_{1}-u_{3}\right)^{2}+\left(\gamma u_{1}^{\prime}-u_{2}\right)^{2}+\left(u_{2}-u_{4}\right)^{2}+u_{3}^{2}+u_{4}^{2}\right)
\end{aligned}
$$

where

$$
K_{b}=\min \left\{1-2 \mu, K_{F B}, K_{F}, k_{B A}-4 \mu, k_{F B}-8 \mu, k_{F}-8 \mu\right\} .
$$

With $K_{b c}=K_{c} / K_{b}$, it follows that $K_{b c} b(u, u) \geq c(u, u)$.

\section{Corollary}

The symmetric bilinear form $b$ is an inner product for $H^{2}$ and $b(u, u) \geq K\|u\|_{H_{2}}^{2}$ for some constant $K$.

The space $H^{1}$ with norm $\|\cdot\|_{W}=\sqrt{c(\cdot, \cdot)}$ induced by the inner product $c$ is called the inertia space and denoted by $W$. It is trivial to see that $\|u\|_{X}^{2} \leq\|u\|_{W}^{2}$ for all $u \in W$ and that $\|\cdot\|_{W}$ and $\|\cdot\|_{H^{1}}$ are equivalent.

The space $H^{2}$ with the norm induced by the inner product $b$ is called the energy space and denoted by $V$. Due to the corollary above it is clear that $\|\cdot\|_{V}$ is equivalent to $\|\cdot\|_{H^{2}}$.

\section{Proposition 4}

If the stability condition holds, then

(a) $V$ is dense in $W$ and $W$ is dense in $X$.

(b) There exist constants $\beta_{1}$ and $\beta_{2}$ such that $\|v\|_{X} \leq \beta_{1}\|v\|_{W} \leq \beta_{2}\|v\|_{V}$ for all $v \in V$.

\section{Proof}

(a) Note that $H^{2}$ is a dense subset of $H^{1}$ and $H^{1}$ is a dense subset of $X$ as $H^{2}(0,1)$ is dense in $H^{1}(0,1)$ and $H^{1}(0,1)$ is dense in $\mathcal{L}^{2}(0,1)$. Now use the equivalence of norms.

(b) The first inequality is trivial and the second is proved in Proposition 3.

Finally we show that the bilinear form $a$ is bounded with respect to the energy norm $\|\cdot\|_{V}$.

\section{Proposition 5}

If the stability condition holds, then there exists a constant $K_{b a}$ such that

$$
|a(u, v)| \leq K_{b a}\|u\|_{V}\|v\|_{V} \text { for each } u \in V \text { and } v \in V .
$$

\section{Proof}

For any $u \in V$,

$$
\begin{aligned}
|a(u, u)|= & C_{F B}\left(\gamma u_{1}-u_{3}\right)^{2}+c_{B A}\left(\gamma u_{1}^{\prime}-u_{2}\right)^{2}+c_{F B}\left(u_{2}-u_{4}\right)^{2} \\
& +C_{F} u_{3}^{2}+c_{F} u_{4}^{2} \\
\leq & K_{a}\left(\left(\gamma u_{1}-u_{3}\right)^{2}+\left(\gamma u_{1}^{\prime}-u_{2}\right)^{2}+\left(u_{2}-u_{4}\right)^{2}+u_{3}^{2}+u_{4}^{2}\right) .
\end{aligned}
$$

In the proof of Theorem 2 it is shown that there exists a constant $K_{b}>0$ such that

$$
\begin{aligned}
b(u, u) & \geq K_{b}\left(\left\|u_{1}^{\prime \prime}\right\|^{2}+\left(\gamma u_{1}-u_{3}\right)^{2}+\left(\gamma u_{1}^{\prime}-u_{2}\right)^{2}+\left(u_{2}-u_{4}\right)^{2}+u_{3}^{2}+u_{4}^{2}\right) \\
& \geq K_{b}\left(\left(\gamma u_{1}-u_{3}\right)^{2}+\left(\gamma u_{1}^{\prime}-u_{2}\right)^{2}+\left(u_{2}-u_{4}\right)^{2}+u_{3}^{2}+u_{4}^{2}\right) .
\end{aligned}
$$


Hence, with $K_{b a}=K_{a} / K_{b}$,

$$
|a(u, u)| \leq K_{b a} b(u, u)=K_{b a}\|u\|_{V}^{2} .
$$

Consequently, as in the standard proof for the Cauchy-Schwartz inequality, $|a(u, v)| \leq \sqrt{a(u, u)} \sqrt{a(v, v)}$, which completes the proof.

\section{$7 \quad$ Proof of the main results}

In this section we apply the existence result from [4]. For convenience we formulate the general linear vibration problem and present the existence theorem. Let $V, W$ and $X$ be real Hilbert spaces with $V \subset W \subset X$. The spaces $X, W$ and $V$ have inner products $(., .)_{X}, c$ and $b$ and norms $\|\cdot\|_{X},\|\cdot\|_{W}$ and $\|\cdot\|_{V}$, respectively.

\section{Problem G}

Find $u$ such that for each $t>0, u(t) \in V, u^{\prime}(t) \in V$ and $u^{\prime \prime}(t) \in W$, and

$$
\begin{gathered}
c\left(u^{\prime \prime}(t), v\right)+a\left(u^{\prime}(t), v\right)+b(u(t), v)=(f(t), v)_{X} \text { for each } v \in V, \\
\text { with } u(0)=u^{\circ} \text { and } u^{\prime}(0)=u^{+} .
\end{gathered}
$$

Theorem [4, Theorem 1]

Assume that

(a) $V$ is dense in $W$ and $W$ is dense in $X$,

(b) there exist constants $\beta_{1}$ and $\beta_{2}$ such that $\|v\|_{X} \leq \beta_{1}\|v\|_{W} \leq \beta_{2}\|v\|_{V}$ for all $v \in V$,

(c) $a(.,$.$) is a non-negative symmetric bounded bilinear form on V$,

(d) $u^{\circ} \in V, u^{+} \in V$ and there exists a $y \in W$ such that

$$
b\left(u^{\circ}, v\right)+a\left(u^{+}, v\right)=c(y, v) \text { for all } v \in V .
$$

Then Problem $\mathrm{G}$ has a unique solution for each $f \in C^{1}([0, \infty), X)$. In addition

$$
u \in C([0, \infty), V) \cap C^{1}((0, \infty), V) \cap C^{1}([0, \infty), W) \cap C^{2}((0, \infty), W) .
$$

The proof of the result relies on the fact that Problem $G$ is equivalent to a system $U^{\prime}=\mathcal{A} U+\mathcal{F}$ in $V \times W$ with initial condition $\left\langle u^{\circ}, u^{+}\right\rangle$. (See proof of the main result in [4].) Conditions (a), (b) and (c) imply that $\mathcal{A}$ is the infinitesimal generator of a $C_{0}$ semigroup. The initial value problem for the first order system has a unique classical solution provided that $\left\langle u^{\circ}, u^{+}\right\rangle \in D(\mathcal{A})$ (otherwise we have merely a mild solution) and this is equivalent to condition (d). The result in [4] contains a typing error; the assumption is that $u^{+} \in V$ as stated above.

\section{Proof of Theorem 1}

The theorem above applies directly to the weak variational form of the hybrid problem due to Propositions 4 and 5.

\section{Proof of Theorem 2}

We use Proposition 1 with $q=0$. For any $g \in C^{1}[0,1]$, we have $\left|g^{\prime}(0)\right| \leq\|g\|_{1}$. Using this estimate and Proposition 1 with $q=0$, we obtain the following result.

$$
\begin{aligned}
& \left\|f_{1}\right\|^{2}+\frac{1}{\alpha}\left\|f_{1}^{\prime}\right\|^{2}+m_{B}\left(f_{1}(0)\right)^{2} \\
\leq & K\left\|u_{1}\right\|_{4}\left\|f_{1}\right\|+K\left\|u_{1}\right\|_{4}\left\|f_{1}^{\prime}\right\|+K\left\|z_{1}\right\|_{2}\left\|f_{1}^{\prime}\right\|+\left(\left|u_{3}\right|+\left|z_{3}\right|\right)\left\|f_{1}^{\prime}\right\| \\
\leq & \frac{K}{2 \varepsilon^{2}}\left(\left\|u_{1}\right\|_{4}^{2}+\left\|z_{1}\right\|_{2}^{2}+u_{3}^{2}+z_{3}^{2}\right)+\frac{K \varepsilon^{2}}{2}\left(\left\|f_{1}\right\|^{2}+\left\|f_{1}^{\prime}\right\|^{2}\right),
\end{aligned}
$$

(C) 2009 European Society of Computational Methods in Sciences and Engineering (ESCMSE) 
for some constant $K$. Choose $\varepsilon$ sufficiently small, then

$$
\left\|f_{1}\right\|_{1}^{2} \leq K\left(\left\|u_{1}\right\|_{4}^{2}+u_{3}^{2}+\left\|z_{1}\right\|_{2}^{2}+z_{3}^{2}\right) .
$$

In Proposition 1 we proved the existence of a mapping $\mathcal{G}$ from $C^{4} \times C^{1}$ into $C$ :

$$
\mathcal{G}\langle u, z\rangle=f .
$$

It is clearly linear. In Proposition 2 we showed that the Green Formula holds for $f, u$ and $z$. The estimate above shows that $\mathcal{G}$ is bounded and hence it can be extended to a linear operator from $H^{4} \times H^{2}$ into $H^{1}$, and (24) remains valid.

\section{References}

[1] A Labuschagne, N F J van Rensburg and A J van der Merwe, Distributed parameter models for a vertical slender structure on a resilient seating, Mathematical and Computer Modelling, 41, (8 - 9) (1021-1033), 2005.

[2] D E Newland, Mechanical vibration analysis and computation, Longman, Essex, 1989.

[3] D E Newland, Calculation of the effect of a resilient seating on the vibration characteristic of slender structures, Engng Struc, 6, 1984, 307-314.

[4] N F J van Rensburg and A J van der Merwe, Analysis of the solvability of linear vibration models, Applicable Analysis, 81(5), (1143-1159), 2002.

\section{Appendix}

\section{Proof of Proposition 1}

Clearly, $f_{2}, f_{3}$ and $f_{4}$ are uniquely defined by (19), (20) and (21). In order to show that $f_{1}$ is uniquely defined, the following boundary value problem for $f_{1}$ is derived from (15) to (18):

$$
\frac{1}{\alpha} f_{1}^{\prime \prime}-f_{1}=u_{1}^{(4)}+\left(\phi u_{1}^{\prime}\right)^{\prime}-q
$$

with

$$
m_{B} f_{1}(0)-\frac{1}{\alpha} f_{1}^{\prime}(0)=-u_{1}^{(3)}(0)-\phi(0) u_{1}^{\prime}(0)-K_{F B}\left(u_{1}(0)-u_{3}\right)-C_{F B}\left(z_{1}(0)-z_{3}\right)
$$

and

$$
\frac{1}{\alpha} f_{1}^{\prime}(1)=u_{1}^{(3)}(1)+\phi(1) u_{1}^{\prime}(1)
$$

Obviously, this boundary value problem has a unique solution and hence $f_{1} \in C^{2}[0,1]$ is defined uniquely.

Next, integration by parts yields

$$
\begin{aligned}
\frac{1}{\alpha}\left\|f_{1}^{\prime}\right\|^{2}= & -\frac{1}{\alpha}\left(f_{1}^{\prime \prime}, f_{1}\right)+\frac{1}{\alpha} f_{1}^{\prime}(1) f_{1}(1)-\frac{1}{\alpha} f_{1}^{\prime}(0) f_{1}(0) \\
= & -\frac{1}{\alpha}\left(f_{1}^{\prime \prime}, f_{1}\right)+\left(u_{1}^{(3)}(1)+\phi(1) u_{1}^{\prime}(1)\right) f_{1}(1) \\
& +\left(-m_{B} f_{1}(0)-u_{1}^{(3)}(0)-\phi(0) u_{1}^{\prime}(0)\right. \\
& \left.-K_{F B}\left(u_{1}(0)-u_{3}\right)-C_{F B}\left(z_{1}(0)-z_{3}\right)\right) f_{1}(0) .
\end{aligned}
$$

(C) 2009 European Society of Computational Methods in Sciences and Engineering (ESCMSE) 
The result now follows from the differential equation by substitution and rearrangement of terms.

\section{Proof of Proposition 2}

For the derivation of the Green formula, multiply (15) by an arbitrary $v_{1} \in C^{2}[0,1]$ and (16) by $v_{1}^{\prime}$. From integration by parts and the boundary conditions (17) and (22), it follows that

$$
\left(f_{1}, v_{1}\right)=-\left(V, v_{1}^{\prime}\right)-V(0) v_{1}(0)+\left(q, v_{1}\right)
$$

and

$$
\frac{1}{\alpha}\left(f_{1}^{\prime}, v_{1}^{\prime}\right)=\left(V, v_{1}^{\prime}\right)-\left(M, v_{1}^{\prime \prime}\right)-M(0) v_{1}^{\prime}(0)+\left(L, v_{1}^{\prime}\right) .
$$

Adding these expressions yields

$$
\left(f_{1}, v_{1}\right)+\frac{1}{\alpha}\left(f_{1}^{\prime}, v_{1}^{\prime}\right)=-\left(M, v_{1}^{\prime \prime}\right)+\left(L, v_{1}^{\prime}\right)-V(0) v_{1}(0)-M(0) v_{1}^{\prime}(0)+\left(q, v_{1}\right) .
$$

This may be written as

$$
c_{A}(f, v)=-b_{A}(u, v)-V(0) v_{1}(0)-M(0) v_{1}^{\prime}(0)+m_{B} f_{1}(0) v_{1}(0)+\left(q, v_{1}\right) .
$$

Now consider Equations (19), (20) and (21). Multiply them by arbitrary constants $v_{2}, v_{3}$ and $v_{4}$ respectively and add to Equation (25). Together with Equations (18) and (23) we have

$$
\begin{aligned}
& c_{A}(f, v)+I_{B} f_{2} v_{2}+m_{F} f_{3} v_{3}+I_{F} f_{4} v_{4} \\
= & -b_{A}(u, v) \\
& -K_{F B}\left(u_{1}(0)-u_{3}\right)\left(v_{1}(0)-v_{3}\right)-C_{F B}\left(z_{1}(0)-z_{3}\right)\left(v_{1}(0)-v_{3}\right) \\
& -k_{B A}\left(u_{1}^{\prime}(0)-u_{2}\right)\left(v_{1}^{\prime}(0)-v_{2}\right)-c_{B A}\left(z_{1}^{\prime}(0)-z_{2}\right)\left(v_{1}^{\prime}(0)-v_{2}\right) \\
& -k_{F B}\left(u_{2}-u_{4}\right)\left(v_{2}-v_{4}\right)-c_{F B}\left(z_{2}-z_{4}\right)\left(v_{2}-v_{4}\right) \\
& -K_{F} u_{3} v_{3}-C_{F} z_{3} v_{3}-k_{F} u_{4} v_{4}-c_{F} z_{4} v_{4}+\left(q, v_{1}\right) .
\end{aligned}
$$

The result follows after presenting Equation (26) in terms of the bilinear forms. 